\title{
Probability tracking of a linear periodic reinforcement schedule'
}

\author{
STEPHEN E. BUGGIE, ${ }^{2}$ San Jose State \\ College, San Jose, Calif. 95114
}

The prediction of Estes' generalized statistical learning theory was verified in a condition in which probability of reinforcement was a noncontingent, linear, periodic function of trials. A strong "probability tracking" effect in which the mean response function approximated the reinforcing event function with a constant tracking "lag" was obtained as expected. An estimate of the stimulus sampling ratio, a parameter of theoretical importance, was obtained from the data.

Numerous studies using the two-choice prediction design originated by Humphreys (1939) have appeared in the literature during recent years. With this design, the phenomenon of "probability learning," in which proportions of responses gradually tend to equal proportions of corresponding reinforcing events, has been demonstrated (e.g., Estes \& Straughan, 1954; Edwards, 1961).

The bulk of probability learning studies have used constant proportions of reinforcing events over trials. This study proposed to test the generality of the statistical learning theory predictions (Estes, 1957) for a condition in which the probability of reinforcement was a linear periodic function of trials. It was expected that the mean response function $\left(P_{n}\right)$ would follow the reinforcement function $\left(\pi_{n}\right)$ fairly closely, but with a constant "lag." Functional interceptions were expected at the maximum and minimum peaks of the response curve with the occurrence of probability tracking behavior (Estes, 1957).

$$
\text { METHOD }
$$

The Ss were 27 naive college student volunteers (mean age $=19.3$ ). Each $S$ was individually run for 300 trials, spaced $5 \mathrm{sec}$ apart. A $2 \times 3 \mathrm{ft}$ three-piece hinged Masonite barrier was placed between the $S$ and the E. A grey metal apparatus box containing batteries, buzzer, and two stimulus event lights was placed before the $S$, and was controlled by the $E$. Noncontingent event schedules were generated from random number tables for each $S$. The period $\mathrm{k}=100$ trials, so the value of $\pi_{n}$ (probability of a given reinforcement occurring on a specified trial $n$ ) can be derived from Eq. 1 .

Eq. I. $\Pi_{n}=\frac{1+(-1)^{k-1}}{2}+\frac{(-1)[n-100(k-1)]}{100}$

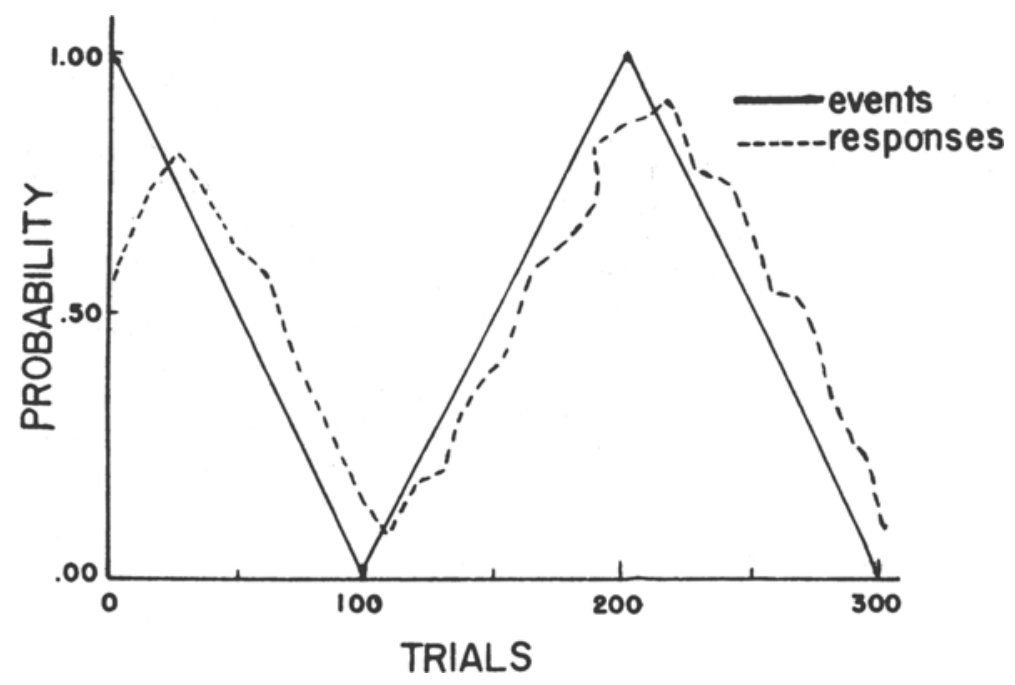

Fig. 1. Probability tracking with a lag over 300 trials with probability of reinforcement as a linear periodic functions of trials.

$\mathrm{k}=$ periodic trial block number (block $=100$ trials).

$\mathrm{n}=$ trial number.

$\pi_{\mathrm{n}}=$ probability of occurrence of a specified reinforcing event on Trial $\mathrm{n}$.

With minor variations due to the statistical derivation of the individual event schedules, the Ss typically found that the two events occurred in a nearly random sequence as $\pi_{n}$ approached .50 around Trials 50,150 , and 250 , but that long sequences of similar reinforcing events would occur as $\pi_{n}$

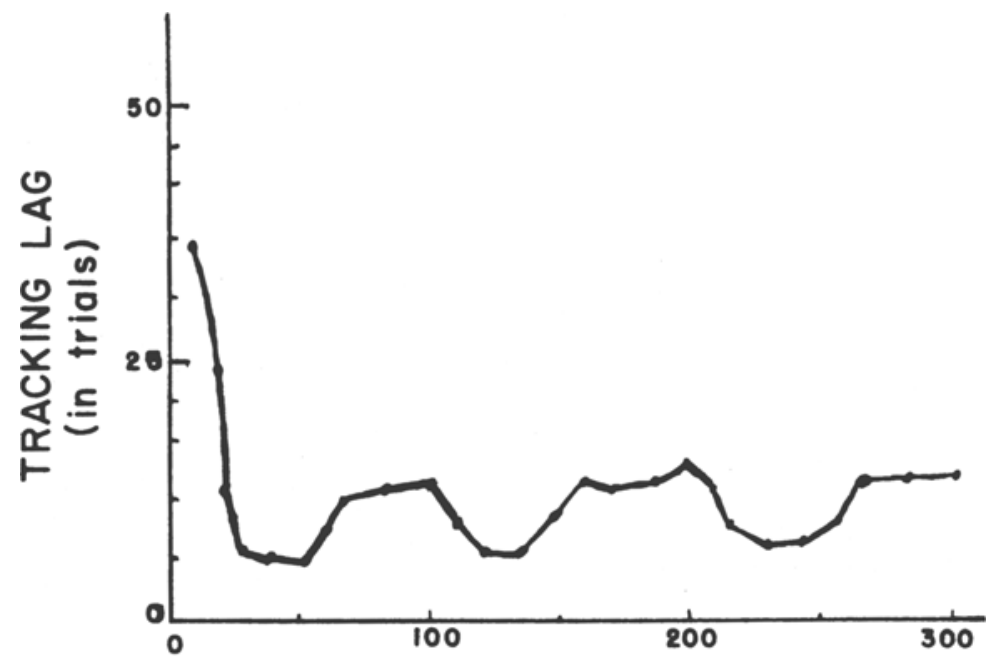

TRIALS deviated greatly from .50 , around Trials 1 , 100,200 , and 300 . The Ss were instructed to predict, on each trial, which of the alternate event lights would occur. When $\pi_{n}$ periodically approached extreme values (i.e., .00 or 1.00 ), sequences of as many as 20 similar events were obtained.

\section{RESULTS AND DISCUSSION}

The obtained results are summarized in Fig. 1.

Following periodic slope reversals of the $\pi_{n}$ function, the $P_{n}$ function intersected the event function at approximately the maximum and minimum points of the 
response function (Fig. 1). Also, inspection of the graph suggested that "probability tracking" occurred as predicted from the generalized statistical learning theory (Estes, 1957, 1959).

The "lag" in probability tracking, a theoretical constant $v$ which was expected to emerge after initial trials, is represented in Fig. 2.

The initial estimate of $v$ from the data was 10.5 trials; however, Estes (1957) implied that this was a biased estimate, since it included lag decrement following the periodic slope reversals of the $\pi_{n}$ function and the subsequent crossover of that function with the $P_{n}$ function. Thus, the apparent periodic decrements in tracking lag around Trials 30,130 , and 230 are actually adjustments for functional slope reversals. Considering this factor, the obtained value of tracking "lag" in Fig. 2 was relatively constant over trials, as expected.

Since $v$ is an inverse function of the stimulus sampling ratio $\Theta$, an estimate of that parameter would yield a more efficient estimate of v. Estes (1957) stated that $\Theta$ can be estimated from the slope of a terminal block of trials of an obtained response function, within a period.

Using Eq. 2, mean estimates of the stimulus sampling ratio $\Theta \cong .074$, and of its inverse, mean tracking lag $v \cong 13.5$ trials, were obtained. These estimates, although not unbiased, were considered adequate for most experimental purposes.

$$
\text { Eq. 2. } \quad \bar{P}_{j}(k, m) \approx a_{j}-\frac{b_{i}}{\theta}+\frac{b_{j}}{2}(2 m k-k+1)
$$

$k=$ number of trials within a block. $\mathrm{m}=$ block within a period.

For this case, $k=50$ trials and $m=2$ (i.e., the second block of 50 trials within each 100-trial period) (from Estes, 1957).

The achievement of the relatively strong probability tracking effect with a stable tracking lag was apparently highly consistent with the predictions of the generalized (Estes, 1957, 1959) statistical learning theory of choice behavior.

\section{REFERENCES}

EDWARDS, W. Probability learning in 1000 trials. Journal of Experimental Psychology, 1961,62, 385-394.

ESTES, W. K. Theory of learning with constant, variable, or contingent probabilities of reinforcement. Psychometrika, 1957, 22, 113-132.

ESTES, W. K. The statistical approach to learning theory. In S. Koch (Ed.), Psychology: A study of a science. Vol. 2. New York: McGraw-Hill 1959. Pp. 380-491.

ESTES, W. K., \& STRAUGHAN, J. H. Analysis of a verbal conditioning situation in terms of statistical learning theory. Journal of Experimental Psychology, 1954, 47, 225-234.

HUMPHREYS, L. G. Acquisition and extinction of verbal expectations in a situation analogou to conditioning. Joumal of Experimental Psychology, 1939, 25, 294-301.

\section{NOTES}

1. A preliminary report of this study was read by the author at the Spartan Psychological Association Meeting, May 20, 1968, San Jose, California

2. Now at the University of Oregon, Eugene Oregon 97403. This study was conducted under the supervision of Professor Robert S. Witte.

\section{Trait similarity and trait evaluation as correlates of attraction}

\begin{abstract}
ABRAHAM TESSER, University of Georgia, Athens, Ga. 30601

The finding that trait similarity and attraction are correlated may be difficult to interpret. Research has indicated that attraction to another is related to the evaluative aspect of the beliefs about Other (i.e., to the extent that Other is believed to have positive attributes, he will be better liked) and that persons tend to attribute positive traits to themselves. To the extent that persons do this, both the similarity and evaluation hypotheses make the same prediction. In assessing the relative importance of each, both were found to make significant contributions to the prediction of attraction with similarity being less important than evaluation.
\end{abstract}

In the social psychological literature, some 0 bearing on those same attributes, one would predict that the more similar $\mathrm{O}$ is to $P$ the more attractive $P$ should find $O$.If, however, in fact, the predicted relationship is found there is some question about its meaning.

Cronbach (1958) has convincingly argued against the uncritical use of dyadic (e.g., similarity) hypotheses. The “... principal difficulty is that interpretations dealing with interactions [e.g., similarity data] can be advanced meaningfully only after the simpler main effects associated with the perceiver or the object of perception have been given separate consideration [p. 355]."

"Consider the hypothesis relating ... [similarity to attraction]. Perhaps ... [attraction] can be predicted directly from the self-perception of . . [P] . . considered alone, perhaps from the perceived characteristics of ... [O] . . . considered alone, or from a linear combination of the two. An interaction hypothesis, i.e., a second-degree relationship, is justified only if it improves significantly on these simpler predictions [p. 356]."

One could plausibly argue that firstdegree relationships can explain the link between trait-similarity and attraction. Let us focus for the moment on P's cognitions about $O$ 's traits. There is a good deal of data indicating that one can predict P's attraction to $O$ just on the basis of the way he evaluates the traits he believes to be charcteristic of $O$. That is, one can sum P's evaluative responses to each of $O$ 's traits or average them in some way and predict his attraction to $\mathrm{O}$ (e.g., Fishbein, 1965).

If it is also true that $P$ tends to attribute positively evaluated traits to himself (Edwards, 1957) then the relationship between $\mathrm{P}-\mathrm{O}$ similarity and P's at traction to $\mathrm{O}$ may be spurious. Take, for example, the limiting case when $\mathbf{P}$ indicates that all the positively evaluated attributes on some list are characteristic of himself and all the negatively evaluated attributes are not. Then, to the extent that $O$ is cognized to have positively evaluated attributes and not to have negatively evaluated attributes he will be more similar to $P$. In this case, both the first-order hypothesis (i.e., evaluation of $O$ 's traits) and the second-order hypothesis (i.e., P-O similarity) would predict more attraction to $O$. If the second-order hypothesis doesn't add any predictive efficiency, Cronbach (1958) would argue that we accept the more parsimonious first-order hypothesis. It is possible and plausible, however, that both sources of variance operate simultaneously and predictive efficiency is maximized when using both.

If the argument is that $P$ bases his attraction toward $O$ on an evaluation of $O$ 's attributes rather than P-O similarity, a viable 